\title{
HOMOGENIZATION OF MONOTONE TYPE PROBLEMS WITH UNCERTAIN DATA
}

\author{
LUDĚK NECHVÁTAL
}

\begin{abstract}
The paper deals with homogenization of nonlinear differential operators with monotone behaviour. We consider a situation, when the coefficients of the operator are not known exactly, but in certain bounds only due to errors caused by measurements. We use the deterministic approach to the problem-worst scenario method introduced by I. Hlaváček.
\end{abstract}

\section{Introduction}

Homogenization is a mathematical method used for modelling of composite materials with periodic structure, where the material properties are described by discontinuous oscillating periodic coefficients that occur in the corresponding differential equations. If the structure is fine, it is almost impossible to solve the problem numerically due to an excessive number of equations resulting from very fine triangulations. Thus, the goal is to replace the original composite material by a fictional homogeneous material, which has the same properties from the macroscopic point of view-i.e., periodic discontinuous coefficients are replaced by constant ones.

In case of exactly given input data (coefficients, right hand side, boundary conditions, etc.) the topic is discussed in many papers and monographs (for introduction see, e.g., [2]), but in practice, considering fixed input parameters is often restrictive. Material constants are usually obtained from experiments by measurements or even from solving inverse (identification) problem. Obviously, both steps are affected by errors, so we are able to prescribe the data in an extent only — we talk about problems with uncertain data.

2000 Mathematics Subject Classification: 35B27, 35 B30.

Keywords: homogenization, uncertain data, worst scenario.

This research was supported by the Grant Agency of the Czech Republic, grant No. 201/08/0874 and the research plan MSM 0021630518 of the Ministry of Education, Youth and Sports of the Czech Republic. 


\section{LUDĚK NECHVÁTAL}

How to solve these problems? According to character of the problem we can follow stochastic or deterministic approach. In stochastic approach the data are considered to be stochastic quantities with certain distribution of probability, which yield stochastic differential equations. Deterministic approach called worst scenario method or method of reliable solution can be used if we want to determine the most danger case, for example if we want to know what data yield the highest temperatures in some crucial places of domain. The main idea consists in defining a special functional over suitable set of admissible data, which can be generally dependent on both data and solution of the problem. In fact, it is a criterion evaluating which data are good or bad. This paper is a continuation of [8], where linear problems were studied, and deals with worst scenario method in homogenization of nonlinear second order boundary value problem of monotone type. For the sake of clarity, we restrict ourselves to problem in dimension one.

The paper is organized into 5 sections. The model problem is introduced in Section 2. Section 3 contains the related homogenization problem. We recall some known results in these two sections, hence, the proofs are omitted. Section 4 is devoted to the worst scenario method including the main result on solvability of the corresponding maximization problem. Some concluding remarks in Section 5 close the paper.

\section{Model problem}

We shall deal with the homogenization problem for a nonlinear second order boundary value problem in an interval. It can be physically interpreted as stationary heat transfer in periodically heterogeneous bar. The coefficient in the equation is supposed to be an uncertain function - it means that the term related to the heat conductivity is known with the given tolerance only. Let $\left\{\varepsilon_{n}\right\}$ be a sequence of small positive parameters converging to zero as $n \rightarrow \infty$. As usual the subscript $n$ is omitted. Homogenization approach deals with the sequence of problems of the same type constructed by the sequence of coefficients with diminishing period. We consider the following model sequence of problems

$$
\begin{aligned}
A_{\varepsilon}\left(u_{\varepsilon}\right) & \equiv-\left[a_{\varepsilon}\left(x, u_{\varepsilon}^{\prime}\right)\right]^{\prime}=f \quad \text { in } I, \\
u_{\varepsilon} & \in W_{0}^{1,2}(I),
\end{aligned}
$$

where $a_{\varepsilon}(x, \xi)=a(x / \varepsilon, \xi), a(y, \xi): \mathbb{R} \times \mathbb{R} \rightarrow \mathbb{R}$ and $a(y+k, \xi)=a(y, \xi), k \in \mathbb{Z}$. Such functions are called $Y$-periodic ( $Y$ is a copy of the periodicity interval, e.g., $Y=[0,1))$. The spaces of $Y$-periodic functions are denoted by $X_{\#}(Y)$. A function $v \in X_{\#}(Y)$ is $Y$-periodic and $v \in X_{\text {loc }}(\mathbb{R})$, i.e., $v \in X(Q)$ for every compact subset $Q \subset \mathbb{R}$. For example, in case $v \in W_{\#}^{1,2}(Y)$, we have $v(0)=v(1)$ 


\section{HOMOGENIZATION OF MONOTONE TYPE PROBLEMS WITH UNCERTAIN DATA}

(such function is continuous on $\mathbb{R}$ due to the compact embedding). The norm of the Banach spaces $X_{\#}(Y)$ is taken as $\|\cdot\|_{X_{\#}(Y)}=\|\cdot\|_{X(Y)}$.

Let the basic period $Y$ consist of $m \in \mathbb{N}$ disjoint intervals $Y_{i}$, i.e., $Y=\bigcup_{i=1}^{m} Y_{i}$, where $Y_{i}=\left[y_{i-1}, y_{i}\right), i=1, \ldots, m$ and let $J=\left[\xi_{1}, \xi_{2}\right]$ be a given closed interval. Moreover, let $\alpha_{i}, c_{1}, c_{2}, C_{L}^{i}, i=1, \ldots, m$ be positive constants. We denote by $S_{\#}$ the set of all functions defined on $Y \times \mathbb{R}$ (and extended by $Y$-periodicity on the whole $\mathbb{R} \times \mathbb{R}$ ) with the following properties:

$$
\begin{array}{crl}
a(y, \xi) \text { is constant in } y \text { on each } Y_{i}, & \xi \in \mathbb{R}, & \\
{[a(y, \xi)-a(y, \eta)](\xi-\eta)>\alpha_{i}|\xi-\eta|^{2},} & \xi, \eta \in J, & y \in Y_{i}, \\
|a(y, \xi)-a(y, \eta)| \leq C_{L}^{i}|\xi-\eta|, & \xi, \eta \in J, & y \in Y_{i}, \\
a(y, \xi)=c_{1}\left(\xi-\xi_{1}\right)+a\left(y, \xi_{1}\right), & \xi<\xi_{1}, \quad y \in Y, \\
a(y, \xi)=c_{2}\left(\xi-\xi_{2}\right)+a\left(y, \xi_{2}\right), & \xi>\xi_{2}, \quad y \in Y .
\end{array}
$$

The assumptions (2)-(5) guarantee that for every function from $S_{\#}$ there exist positive constants $\alpha$ and $C_{L}$ such that

$$
\begin{array}{rlrl}
{[a(y, \xi)-a(y, \eta)](\xi-\eta)} & \geq \alpha|\xi-\eta|^{2}, & & \xi, \eta, y \in \mathbb{R} \\
|a(y, \xi)-a(y, \eta)| & \leq C_{L}|\xi-\eta|, & \xi, \eta, y \in \mathbb{R} .
\end{array}
$$

The set $\mathscr{U}^{a d}$ of admissible coefficients is defined by

$$
\mathscr{U}^{a d}=\left\{a \in S_{\#}: a_{\min }(y, \xi) \leq a(y, \xi) \leq a_{\max }(y, \xi)\right\},
$$

where $a_{\min }<a_{\max }$ (bounds of measurements) are given functions from $S_{\#}$.

The properties (6) and (17) ensure the strong monotonicity and Lipschitz continuity of the operator $A_{\varepsilon}$. These two properties guarantee existence and uniqueness of the solution (the proof is based on the fixed point theorem and can be found, e.g., in [4]). More precisely, the following holds:

Theorem 1. Let $a \in \mathscr{U}^{a d}$. Then there exists the unique solution $u_{\varepsilon}(a) \in W_{0}^{1,2}(I)$ of the problem (11) for every $f \in L^{2}(I)\left(f \in L^{1}(I)\right.$ can be admitted due to the compact embedding). Moreover, we have an apriori estimate $\left\|u_{\varepsilon}\right\|_{W_{0}^{1,2}(I)} \leq c$, where constant $c$ does not depend on $\varepsilon$.

Although the existence and uniqueness of the solution can be obtained also under weaker monotonicity and continuity assumptions, we need the introduced properties in what follows.

\section{Homogenized problem}

There are several ways how to handle the homogenized problem. Besides the asymptotic expansion method [2], $G, H$ and $\Gamma$-convergence [5], [7, the two-scale 


\section{LUDĚK NECHVÁTAL}

convergence method [1] seems to be the most powerful tool in homogenization theory. Using one of these concepts we obtain the following homogenization result related to problem (11):

Theorem 2. Let $a \in \mathscr{U}^{a d}$ and let $u_{\varepsilon}(a)$ be the solution of the problem (11). Then we have

$$
\begin{aligned}
u_{\varepsilon} & \rightarrow u_{0} & & \text { in } W_{0}^{1,2}(I), \\
a_{\varepsilon}(x, \xi) & \rightarrow b(\xi) & & \text { in } L^{2}(I),
\end{aligned}
$$

where $u_{0}(a)$ is unique solution of the homogenized problem

$$
\begin{aligned}
A_{0}\left(u_{0}\right) & \equiv-\left[b\left(u_{0}^{\prime}\right)\right]^{\prime}=f \quad \text { in } I, \\
u_{0} & \in W_{0}^{1,2}(I) .
\end{aligned}
$$

Coefficient $b: \mathbb{R} \rightarrow \mathbb{R}$ is defined by

$$
b(\xi)=\int_{Y} a\left(y, \xi+w_{\xi}^{\prime}(y)\right) \mathrm{d} y,
$$

where function $w_{\xi}$ is the unique solution of the local problem

$$
\begin{gathered}
-\left[a\left(y, \xi+w_{\xi}^{\prime}(y)\right)\right]^{\prime}=0, \\
w_{\xi} \in W_{\# 0}^{1,2}(Y)
\end{gathered}
$$

(subscript " 0 " denotes functions with zero mean value over $Y$ ). Moreover, coefficient $b(\xi)$ satisfies $\forall \xi, \eta \in \mathbb{R}$ the following estimates

$$
\begin{aligned}
{[b(\xi)-b(\eta)](\xi-\eta) } & \geq \alpha|\xi-\eta|^{2}, \\
|b(\xi)-b(\eta)| & \leq C_{L}|\xi-\eta|,
\end{aligned}
$$

where $\alpha, C_{L}$ are the same constants as in (6) and (7).

Let us note that in case of higher dimension the estimate (12) becomes generally

$$
|b(\xi)-b(\eta)| \leq \frac{C_{L}^{2}}{\alpha}|\xi-\eta|
$$

In literature the additional assumption $a(y, 0)=0$ is usually used which simplifies proofs a bit. Otherwise, we can follow the proof presented in [3]. Some other variants of monotonicity assumptions have also been studied.

\section{Worst scenario method}

In this section we define the objective functional over the set $\mathscr{U}^{a d}$ and formulate the corresponding maximization problem. Let us consider simple case 
when we are interested in maximum of the homogenized solution (temperature) in a subinterval $\widetilde{I} \subset I$ (usually a crucial place in the material). Thus, the functional $\Phi$ is defined by

$$
\Phi(a)=\frac{1}{|\widetilde{I}|} \int_{\widetilde{I}} u_{0}(a) \mathrm{d} x
$$

(average value of homogenized solution $u_{0}$ over $\widetilde{I}$ which is dependent on the coefficient $a \in \mathscr{U}^{a d}$ ) and the worst scenario problem reads:

$(\operatorname{Max} \mathbf{P})$. Find $\widehat{a} \in \mathscr{U}^{a d}$ such that $\Phi(a) \leq \Phi(\widehat{a}), \forall a \in \mathscr{U}^{a d}$.

Before formulation of existence theorem to problem (MaxP) we introduce the two useful lemmas:

LeMma 1. Every sequence of functions $\left\{a_{n}\right\} \subset \mathscr{U}^{\text {ad }}$ contains an uniformly convergent subsequence, i.e., there exists an element $a \in \mathscr{U}^{a d}$ such that $a_{n^{\prime}} \rightrightarrows a$ in $Y \times \mathbb{R}$.

Proof. Let $\mathscr{U}_{J}^{a d}$ denote the set of restricted admissible functions to interval $J$. Since the function $a \in \mathscr{U}_{J}^{a d}$ can be identified with $m$ one-variable functions which are Lipschitz on $J$, Arselà-Ascoli theorem yields that the set $\mathscr{U}_{J}^{a d}$ is compact in $[C(J)]^{m}$, i.e., from every sequence $\left\{a_{n}\right\}$ it is possible to extract a subsequence $\left\{a_{n^{\prime}}\right\}$ which converges uniformly to an element $a \in \mathscr{U}_{J}^{a d}$. Further, since the set $\mathscr{U}^{a d}$ is the continuous extension of the set $\mathscr{U}_{J}^{a d}$ by lines with the same slope, the uniform convergence in the whole $\mathbb{R}$ follows immediately.

Lemma 2. Let $\left\{a_{n}\right\} \subset \mathscr{U}^{a d}$ be a sequence such that $a_{n} \rightrightarrows a$ in $Y \times \mathbb{R}$. Then $u_{0}\left(a_{n}\right) \rightarrow u_{0}(a)$ in $W_{0}^{1,2}(I)$, where $u_{0}\left(a_{n}\right), u_{0}(a)$ are the solutions of the problem (8) with coefficient $a_{n}$ and $a$, respectively.

Proof. Let us denote $w_{n}:=w_{\xi}\left(a_{n}\right)$ and $w:=w_{\xi}(a)$ the solutions of the local problem (10) with the coefficient $a_{n}$ and $a$, respectively. First, let us prove the boundedness of the sequence $w_{n}$. From Lipschitz continuity (7) we have

$$
|a(y, \xi)|-|a(y, 0)| \leq|a(y, \xi)-a(y, 0)| \leq C_{L}|\xi| \Rightarrow|a(y, \xi)| \leq c+C_{L}|\xi|
$$

for all $a \in \mathscr{U}^{a d}$, where $c=\max \left\{\max _{y \in Y}\left|a_{\max }(y, 0)\right|, \max _{y \in Y}\left|a_{\min }(y, 0)\right|\right\}$. Using strong monotonicity condition ([6), the weak formulation of the problem (10) and 


\section{LUDĚK NECHVÁTAL}

the inequality $|a b| \leq \frac{1}{2 \theta} a^{2}+\frac{\theta}{2} b^{2}$, we may write

$$
\begin{aligned}
\alpha\left\|\xi+w_{n}^{\prime}\right\|_{L_{\#}^{2}(Y)}^{2} & \leq\left|\int_{Y}\left[a_{n}\left(y, \xi+w_{n}^{\prime}\right)-a_{n}(y, 0)\right]\left(\xi+w_{n}^{\prime}\right) \mathrm{d} y\right| \\
& =\left|\xi \int_{Y} a_{n}\left(y, \xi+w_{n}^{\prime}\right) \mathrm{d} y-\int_{Y} a_{n}(y, 0)\left(\xi+w_{n}^{\prime}\right) \mathrm{d} y\right| \\
& \leq|\xi| \int_{Y}\left|a_{n}\left(y, \xi+w_{n}^{\prime}\right)\right| \mathrm{d} y+c \int_{Y}\left|\xi+w_{n}^{\prime}\right| \mathrm{d} y \\
& \leq|\xi| c+\left(|\xi| C_{L}+c\right) \int_{Y}\left|\xi+w_{n}^{\prime}\right| \mathrm{d} y \\
& \leq|\xi| c+\frac{\theta}{2}\left(|\xi| C_{L}+c\right)^{2}+\frac{1}{2 \theta}\left\|\xi+w_{n}^{\prime}\right\|_{L_{\#}^{2}(Y)}^{2} .
\end{aligned}
$$

Overall

$$
\left(\alpha-\frac{1}{2 \theta}\right)\left\|\xi+w_{n}^{\prime}\right\|_{L_{\#}^{2}(Y)}^{2} \leq|\xi| c+\frac{\theta}{2}\left(|\xi| C_{L}+c\right)^{2},
$$

where $\theta$ is a suitably large constant. Hence, with help of inequality $|x| \leq \mid x+$ $y|+| y \mid$ and Poinacaré inequality, we see that $\left\|w_{\xi}\left(a_{n}\right)\right\|_{W_{\# 0}^{1,2}(Y)} \leq c_{\xi}$. It means that there exists an element $\omega_{\xi} \in W_{\# 0}^{1,2}(Y)$ such that, up to a subsequence, $w_{n^{\prime}} \rightarrow \omega$ in $W_{\# 0}^{1,2}(Y)$. The uniform convergence $a_{n} \rightrightarrows a$ in $Y \times \mathbb{R}$ implies the strong convergence $a_{n} \rightarrow a$ in $L_{\#}^{2}(Y)$ for every $\xi \in \mathbb{R}$. Using again (6) we have

$$
\begin{aligned}
& \alpha\left\|w_{n^{\prime}}^{\prime}-w^{\prime}\right\|_{L_{\#}^{2}(Y)} \\
& \leq\left|\int_{Y}\left[a_{n^{\prime}}\left(y, \xi+w_{n^{\prime}}^{\prime}\right)-a_{n^{\prime}}\left(y, \xi+w^{\prime}\right)\right]\left(w_{n^{\prime}}^{\prime}-w^{\prime}\right) \mathrm{d} y\right| \\
& =|\underbrace{}_{=0} a_{n^{\prime}}\left(y, \xi+w_{n^{\prime}}^{\prime}\right)\left(w_{n^{\prime}}^{\prime}-w^{\prime}\right) \mathrm{d} y-\int_{Y} a_{n^{\prime}}\left(y, \xi+w^{\prime}\right)\left(w_{n^{\prime}}^{\prime}-w^{\prime}\right) \mathrm{d} y| \\
& =\left|\int_{Y} a_{n^{\prime}}\left(y, \xi+w^{\prime}\right)\left(w_{n^{\prime}}^{\prime}-\omega^{\prime}\right) \mathrm{d} y+\int_{Y} a_{n^{\prime}}\left(y, \xi+w^{\prime}\right)\left(\omega^{\prime}-w^{\prime}\right) \mathrm{d} y\right| .
\end{aligned}
$$

The first integral on the right-hand side converges to zero, since it contains the product of strongly and weakly converging sequence in $L_{\#}^{2}(Y)$. The second integral converges to $\int_{Y} a\left(y, \xi+w^{\prime}\right)\left(\omega^{\prime}-w^{\prime}\right) \mathrm{d} y$ which equals to zero due to the definition of solution $w$. Hence, we proved that $w_{n^{\prime}}^{\prime} \rightarrow w^{\prime}$ in $L_{\#}^{2}(Y)$. On the 
other hand, we know that $w_{n^{\prime}}^{\prime} \rightarrow \omega^{\prime}$ in $L_{\#}^{2}(Y)$ so that by uniqueness of the limit we have $w^{\prime}=\omega^{\prime}$ a.e. in $L_{\#}^{2}(Y)$. Since $w$ is the unique weak solution of the problem (10), the entire sequence converges.

Now we can proceed to the proof of uniform convergence of the sequence of homogenized coefficients $b_{n}(\xi)$. By definition (9) we have

$$
\begin{aligned}
\sup _{\xi \in \mathbb{R}}\left|b_{n}(\xi)-b(\xi)\right|= & \sup _{\xi \in \mathbb{R}}\left|\int_{Y}\left[a_{n}\left(y, \xi+w_{n}^{\prime}\right) \mathrm{d} y-a\left(y, \xi+w^{\prime}\right)\right] \mathrm{d} y\right| \\
\leq & \sup _{\xi \in \mathbb{R}} \int_{Y}\left|a_{n}\left(y, \xi+w_{n}^{\prime}\right) \mathrm{d} y-a_{n}\left(y, \xi+w^{\prime}\right)\right| \mathrm{d} y \\
& +\sup _{\xi \in \mathbb{R}}\left|\int_{Y}\left[a_{n}\left(y, \xi+w^{\prime}\right) \mathrm{d} y-a\left(y, \xi+w^{\prime}\right)\right] \mathrm{d} y\right| \\
\leq & C_{L} \int_{Y}\left|w_{n}^{\prime}-w^{\prime}\right| \mathrm{d} y \\
& +\sup _{\xi \in \mathbb{R}}\left|\int_{Y}\left[a_{n}\left(y, \xi+w^{\prime}\right) \mathrm{d} y-a\left(y, \xi+w^{\prime}\right)\right] \mathrm{d} y\right| .
\end{aligned}
$$

The first integral on the right-hand side converges to zero thanks to the strong convergence $w_{n}^{\prime} \rightarrow w^{\prime}$ in $L_{\#}^{2}(Y)$ and the second integral converges to zero due to the uniform convergence of the sequence of coefficients $a_{n}$. It means that $b_{n}(\xi) \rightrightarrows b(\xi)$ in $\mathbb{R}$.

Finally, let us denote by $u_{n}:=u_{0}\left(a_{n}\right)$ and $u:=u_{0}(a)$ the weak solutions of the homogenized problem (8). In similar way as above, we show with help of property (11) that the sequence of solutions $u_{n}$ is bounded, i.e.,

$$
\begin{aligned}
\alpha\left\|u_{n}^{\prime}\right\|_{L^{2}(I)}^{2} & \leq\left|\int_{I}\left[b_{n}\left(u_{n}^{\prime}\right)-b_{n}(0)\right] u_{n}^{\prime} \mathrm{d} x\right| \\
& =\left|\int_{I} f u_{n} \mathrm{~d} x-\int_{I} b_{n}(0) u_{n}^{\prime} \mathrm{d} x\right| \\
& \leq\left(M\|f\|_{L^{2}(I)}+c\right)\left\|u_{n}^{\prime}\right\|_{L^{2}(I)},
\end{aligned}
$$

where $M$ is a constant from Friedrich's inequality and $c$ is a bound of coefficients $b_{n}$ at the point 0 . It proves that solutions $u_{n}$ are bounded in $W_{0}^{1,2}(I)$ and thus there exists an element $\widetilde{u} \in W_{0}^{1,2}(I)$ such that, up to a subsequence $u_{n^{\prime}} \rightarrow \widetilde{u}$ in $W_{0}^{1,2}(I)$. It remains to prove that $u^{\prime}=\widetilde{u}^{\prime}$ a.e. in $L^{2}(I)$ which can be shown 


\section{LUDĚK NECHVÁTAL}

in the same way as in case of sequence of solutions $w_{n^{\prime}}$. Due to the uniqueness of the limit, the entire sequence converges and the proof is complete.

Now we are in position to formulate our main result.

Theorem 3. There exists a solution of the problem (MaxP).

Pr o of. Let $\left\{a_{n}\right\} \subset \mathscr{U}^{a d}$ be a maximizing sequence of the functional $\Phi$, i.e.,

$$
\lim _{n \rightarrow \infty} \Phi\left(a_{n}\right)=\sup _{a \in \mathscr{U}^{a d}} \Phi(a) .
$$

From Lemma 1 we know that there exists an element $\widetilde{a} \in \mathscr{U}^{a d}$ such that, up to a subsequence,

$$
a_{n^{\prime}} \rightrightarrows \widetilde{a} \quad \text { in } \quad Y \times \mathbb{R}
$$

Lemma 2 yields $u_{0}\left(a_{n^{\prime}}\right) \rightarrow u_{0}(\widetilde{a})$ in $W_{0}^{1,2}(I)$, and as a consequence of definition of functional $\Phi$,

$$
\Phi\left(a_{n^{\prime}}\right) \rightarrow \Phi(\widetilde{a}) \quad \text { as } \quad n^{\prime} \rightarrow \infty .
$$

Combining (13) and (14) we get

$$
\lim _{n^{\prime} \rightarrow \infty} \Phi\left(a_{n}^{\prime}\right)=\Phi(\widetilde{a})=\sup _{a \in \mathscr{U}^{a d}} \Phi(a)
$$

and thus $\widetilde{a}$ is a maximizing element, i.e., $\widetilde{a}=\widehat{a}$ in $Y \times \mathbb{R}$, which is the desired result.

\section{Concluding remarks}

We demonstrated the usage of worst scenario method in homogenization of nonlinear boundary value elliptic problem with uncertain coefficient. For the sake of brevity we restricted ourselves to the problem in dimension one. The simple objective functional was considered. It can be interpreted as the average temperature over some crucial place of material and the corresponding worst scenario problem investigates the most "danger" data that yield the highest temperature. According to the technical requirements, various functionals can be considered. On the other hand, these functionals must satisfy some minimal conditions introduced in [6]. Once the worst scenario problem is set, the further steps would follow:

i) The formulation of the approximate worst scenario problem-it requires a discretization in both the space, where the solution of the model problem is looked for, and the set of admissible data.

ii) The convergence analysis of the approximate solutions to the exact solution. 


\section{HOMOGENIZATION OF MONOTONE TYPE PROBLEMS WITH UNCERTAIN DATA}

iii) Proposal of suitable computer code.

These steps can be completed by sensitive analysis with respect to input data, see [6].

\section{REFERENCES}

[1] ALLAIRE, G.: Homogenization and two-scale convergence, SIAM J. Math. Anal. 23 (1992), 1482-1518.

[2] Bensoussan, A.-LIOns, J. L.-PAPAniCOlaOU, G.: Asymptotic Analysis for Periodic Structures. Stud. Math. Appl., Vol. 5, North Holland, Amsterdam, 1978.

[3] CHIADÒ PIAT, V.-DEFRANCESCHI, A.: Homogenization of monotone operators, Nonlinear Anal. 14 (1990), 717-732.

[4] FRANCÜ, J.: Monotone operators. A survey directed to applications to differential equations, Appl. Math. 35 (1990), 256-301.

[5] DE GIORGI, E.-SPAGNOLO, S.: Sulla convergenza degli integrali dell'energia per operatori ellittici del secondo ordine, Boll. Un. Mat. Ital., IV. Ser. 8 (1973), 391-411.

[6] HLAVÁČEK, I.-CHLEBOUN, J.-BABUŠKA, I.: Uncertain Input Data Problems and the Worst Scenario Method. Appl. Math. Mech. (North-Holland), Vol. 46, Elsevier, Amsterdam, 2004.

[7] MURAT, F.-TARTAR, L.: H-convergence, Topics in Mathematical Modelling of Composite Materials. Progr. Nonlinear Differential Equations Appl., Vol. 31, Birkhauser, Boston, MA, 1997.

[8] NECHVÁTAL, L.: Worst scenario method in homogenization, Appl. Math. 51 (2006), 263-294.

Received September 29, 2008
Institute of Mathematics

Faculty of Mechanical Engineering

Brno University of Technology

Technická 2

CZ-616-69 Brno

CZECH REPUBLIC

E-mail: nechvatal@fme.vutbr.cz 